\title{
Thermal NDE of thick GRP panels by means of a Pulse Modulated Lock-In Thermography technique
}

\author{
G. Pitarresi ${ }^{1 \text {,a }}$ \\ ${ }^{1}$ University of Palermo, Dipartimento di Meccanica, Palermo, Italy
}

\begin{abstract}
This work describes the development and implementation of an infrared thermal NDE procedure for the evaluation of subsurface defects. The approach is called Pulse-Modulated Lock-In Thermography (PMLT) and is based on the analysis of the frequency response of the measured temperature and comparison with the carrier frequencies launched by the external heat delivering source. The heat deposited on the object is in particular modulated as a train of square waves. This is easily achieved by periodically shuttering the heat source. The temperature is then sampled throughout the deposition of a few square waves. A lock-in algorithm is then implemented able to selectively filter out components at the main carrier frequencies of the heating signal and evaluate the phase information. Defected areas at different depths can be marked based on phase contrast, by using data from a single experiment as in Pulsed-Phase Thermography. An artificially defected thick GRP panel typical of naval monolithic hull structures, is investigated to validate the proposed technique. Experimental data have confirmed the potentials of PMLT as a flexible IR NDT approach, and its ability to be implemented by means of low cost heating and IR equipments.
\end{abstract}

\section{Introduction}

The non-contact full-field measuring features of modern IR thermocameras, and their latest progresses in sampling rates, thermal and geometric resolution have all contributed to significantly foster interest towards thermal methods in general [1]. Application of thermal methods for material structural assessment comprise Infrared Thermography for Non-Destructive Testing (IRNDT or TNDE for thermal NDE [2]). This is mostly based on the Active Thermography approach according to which an external stimulation (thermal or mechanical) is delivered to the structure and the thermal response enquired to find defect signatures for qualitative or quantitative defect evaluation. A number of IRNDT techniques are now available which are finding successful application [3], probably unrivalled when fast full field evaluation of shallow depths in complex non homogeneous FRP composite materials is required. Most of the available IRNDT techniques can be distinguished as belonging to one of the following two main approaches: transient or modulated thermography [3].

In transient thermography the thermal decay transitory (i.e. the cooling transitory) following the external stimulation is sampled on the component surface. When external heat sources are used, these usually consist in high power optical lamps able to deposit a very intense and short heat

\footnotetext{
a e-mail : pitarresi@dima.unipa.it
} 
impulse on the component surface [2]. Several applications have been proposed based on the evaluation of thermal contrast between a defected and a sound area [2-6]. A common drawback of these techniques is the opportune choice of a reference sound area when, as usually happens, a non uniform heat deposition is present. The thermal decay of a sound area is by itself strongly dependent on the local quantity of heat received, so retrieval of defect quantitative data becomes very difficult due to the strong influence of the position of the sound area used in the calculation. Some clever solutions have been proposed in order to avoid the above influence. The recent Source Distribution Image (SDI) proposes a criterion for choosing the sound area on zones which have received the same amount of heat [4]. The TSR [5] and DAC [6] techniques both are based on synthetic data reconstruction based on the expected, rather than measured, features of the sound area thermal decay. All methods still require a careful control of the heating pulse intensity and duration. In fact heat accumulation on the defected area should start only after heat deposition, i.e. the temperature measured at the early stages of the cooling transitory should still remain unaffected by the presence of a subsurface defect. Based on the evaluation of the cooling transitory is also Pulsed Phase Thermography (PPT) $[7,8]$. In this case the temperature is sampled over a truncated time window, after a Dirac heat impulse deposition, and the Discrete Fourier Transform (DFT) is then performed on the acquired signal. A shift in time is observed on the harmonics of the measured signal between defected and sound areas. Furthermore the extent of these phase shifts is dependent on defect depth and material thermal properties. The phase shift information at the different frequencies can be retrieved by a DFT analysis on the sampled signal and the phase contrast between defected and sound areas exploited to investigate defects.

Modulated or Lock-In Thermography (LT) $[9,10]$ has many similarities with PPT. In fact in LT a phase shift between defected and sound areas is observed on the measured harmonic response of the component, and this phase contrast represents again the defect signature. Two main differences between PPT and LT arise though:

- in LT the temperature is sampled in a stationary regime during which heat is continuously deposited following a periodic law having a main carrier frequency (usually a sinusoidal law);

- only the harmonic response having the same frequency of the carrier $f_{c}$ is considered, so that only a single value of phase shift is obtained per each sampled point and per each experiment. In order to explore the response at different frequencies a new experiment has to be performed each time with a new value of $f_{c}$.

The first feature of LT, as outlined above, gives some advantages over the PPT technique: in fact the modulation of heat deposition with a single carrier frequency concentrates much of the power spectrum on this frequency value and this allows the possibility to use heat sources at lower power, although some means for modulating the delivered heat are required. Furthermore sampling windows containing a multiple number of signal periods can reduce the influence of noise (e.g. leakage or ripple [3]) and the lock-in correlation between pure sinusoidal waves is straight forward in terms of signal processing $[9,10]$. The second feature represents a drawback compared to PPT since the measured phase shift at the given carrier frequency might not be the optimised value at different defect depths [11]. A theoretical explanation of this behaviour is provided by considering the dependence between the carrier frequency $f_{c}$ and the diffusion length parameter, which is a damping parameter present in the solution of the Fourier law for the temperature distribution on a semi-infinite homogeneous medium under a stationary sinusoidal heating of the surface $[3,12,13]$. This relationship states that diffusion length increase with decreasing $f_{c}$. So in LT it will be necessary to perform different experiments at varying $f_{c}$ to probe at different depths. A common advantage of PPT, LT and techniques based on phase contrast in general, over techniques based on thermal contrast, is a reduced influence to the following important issue: non uniform heat deposition on the analysed area, non-homogeneous emissivity of the component surface, heat reflection from the environment, curvature of the scanned component surface [3].

In this study a new approach is proposed in terms of heat deposition and lock-in signal processing, which is believed to maintain some of the advantages peculiar to PPT or LT, while also simplifying the hardware requirements and hence the implementation set-up. This approach will be 
referred to as Pulse-Modulated Lock-In Thermography (PMLT) and consists in a square wave modulation of the heat delivered to the component. A suitable lock-in filtering procedure, easy to implement via software, is also presented in order to selectively perform the lock-in correlation on the harmonics at different frequencies contained in the sampled signal. The whole procedure is implemented through the use of low cost heating and IR hardware, and used to investigate the detectability of deep defects in a thick glass fibre reinforced panel. Some experimental results are presented which confirm the validity of the assumptions and the potentials of the PMLT scheme.

\section{Pulse-Modulated Lock-In Thermography (PMLT)}

The PMLT approach proposed in this work implements the typical LT scheme, where the sampling of thermograms is performed during the stationary regime of a modulated external heating. In LT the usual modulation employed is through a monofrequence sinusoidal signal. In PMLT the heat deposition is modulated by the succession of square waves. If the external heat source is provided by optical stimulation, this can be easily obtained and controlled by just periodically shattering the heat path to the component.

Compared to the PPT, the pulse modulation will deliver a major power quantity at the main carrier frequency, while compared to the LT, the square wave will as well deliver harmonics at higher frequency which can probe the component at progressively shallower depths. This will result in the possibility to probe a wider range of depths with only one experiment with an initial opportune tuning of the pulse-modulation parameters (see section 2.1). By extending the sampling window to a slightly increased number of square wave periods delivered to the component will also concentrate the thermal power on a reduced number of harmonics, allowing the use of low power lamps (e.g. halogen lamps) than those used in PPT. With low thermal diffusivity materials, such as FRP composites, the duration of each heat impulse will generally extend to many seconds, in order to obtain a sufficiently low value of the main carrier frequency $f_{c}$. This results in a less restricted need to carefully control the heat impulse duration.

\subsection{Influence of operational parameters in PMLT}

In order to evaluate the potentials of PMLT it is essential to relate the modulation parameters defining the train of square pulses with the power spectrum of heat delivered, i.e. with the power associated to each delivered harmonic. In order to do so the Discrete Fourier Transform (DFT) is used to investigate the frequency content of a simulated, discretely sampled, sequence of square waves. The power spectrum, i.e. the absolute value of the DFT of the signal, is derived by using the $M_{a t l a b}{ }^{\circledR} f f t$ function and plotted under various conditions (see fig. 1,2). The principal modulation parameters $(T, n p, I)$ and the relationship with the time and frequency parameters governing the problem are defined in table 1.

Figures 2a-e analyse the harmonic content change when an integer number of square wave periods is considered within the time window. It is possible to observe always the presence of harmonics with higher power alternating groups of harmonics with lower power with some regular progression. These power-peak harmonics will be called carriers and, principal carrier will be the one with the lowest nonzero frequency $(k=1)$, while all others will be referred to as secondary carriers $(k>1)$.

In fig. 2a the influence of the number of periods is evidenced for three values of $n p$ and fixed sampling time. A smaller $n p$ is able to deliver heat at lower carrier frequencies, and increase also the number of carriers, although the carriers power decrease more with increasing frequency.

In figure $2 b, c$ the influence of the pulse amplitude is investigated. In [14] a slight time shift in the impulse delivery of two flash lamps allowed to perform a PPT analysis comparing two cases: heat delivered in one impulse, and heat delivered by two successive impulses of half amplitude. Since two successive impulses are already able to introduce a principal carrier with higher power it came out that the loss of impulse amplitude had little influence on the results. This mechanism is the same 
observed in PMLT where the sequence of square waves increases the power in the principal and in the secondary carriers compared to what is able to deliver at the same frequencies a single pulse of equal intensity over the same time window.

Table 1. definition of discrete signal processing parameters.

\begin{tabular}{|c|c|c|c|c|}
\hline \multicolumn{2}{|c|}{ Parameter } & Relationships & simulation & Experimental \\
\hline$I_{s}$ & Heat deploying signal & $/$ & & \\
\hline$T$ & Sampling window & $T=N \cdot T s$ & $1,2,10$ & $480 \mathrm{~s}$ \\
\hline$n p$ & Number of square waves in $T$ & $/$ & $1,3,4,5,10$ & 3 \\
\hline$I$ & Square wave amplitude & $/$ & $1,2,5$ & \\
\hline$N$ & Number of samples & $/$ & & 392 \\
\hline$i$ & Sampling index & $i=1, \ldots, N+1$ & & \\
\hline$T_{s}$ & Sampling time interval & $T s=T / N$ & $0.0005,0.001$ & $1.22 \mathrm{~s}$ \\
\hline$t$ & Time domain & $(i-1) \cdot T s$ & & \\
\hline$f_{s}$ & Sampling frequency & $1 / T_{s}$ & & $0.82 \mathrm{~Hz}$ \\
\hline$\Delta f$ & Fundamental frequency & $1 / T=1 /\left(N T_{s}\right)$ & & $0.0021 \mathrm{~Hz}$ \\
\hline$f_{c}$ & Principal carrier frequency & $n p / T$ & & $0.00625 \mathrm{~Hz}$ \\
\hline$f_{N}$ & Maximum (Nyquist) frequency & $1 /\left(2 T_{s}\right)$ & & $0.41 \mathrm{~Hz}$ \\
\hline$w_{p}$ & Principal carrier pulsation & $n p \cdot(2 \pi / T)=2 \pi \cdot f_{c}$ & & $w_{l}=0.039 \mathrm{rad} / \mathrm{s}$ \\
\hline$k$ & Secondary carrier frequency index & $/$ & & \\
\hline$w_{k}$ & Secondary carrier pulsation & $w_{k-1}+2 \cdot w_{p}$ & & $w_{2}=0.119 \mathrm{rad} / \mathrm{s}$ \\
\hline$F_{k}$ & $k_{t h}$ in-phase reference signal & $\operatorname{Sin}\left(w_{k} \cdot t\right)$ & & \\
\hline$G_{k}$ & $k_{t h}$ in-quadrature reference signal & $\operatorname{Cos}\left(w_{k} \cdot t\right)$ & & \\
\hline
\end{tabular}
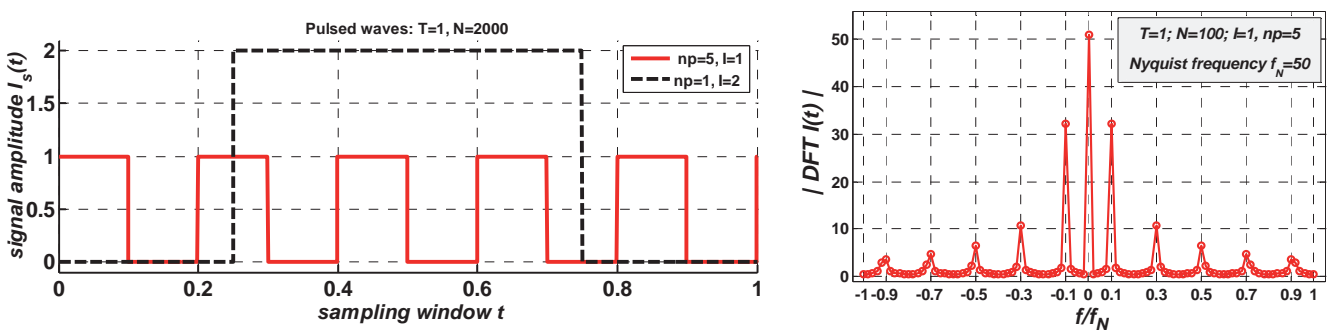

Fig. 1. Left) trains of square waves with equal high and low durations. Right) Example of a power spectrum computed with the fft Matlab ${ }^{\circledR}$ function.

Figure $2 b$ in particular shows that the power of the carriers for a sequence of $n p=5$ periods is the same as that of one single impulse with an intensity five times higher, when both are deployed over the same time window. The number of carriers is smaller for the repeated impulse. Figure $2 \mathrm{c}$ shows that a single impulse 10 times more intense than ten repeated impulses deploys the same carrier frequencies and the same carriers power if the duration of the period of the two signals is the same. This means that the time window of the signal $I_{s}(n p=10)$ will be 10 times longer than $I_{s}(n p=1)$. This increase in time though is not necessarily a drawback for PMLT compared to PPT. In fact in PMLT the temperature is sampled during heat delivery, while in PPT is sampled after the delivery of a short heat impulse, and sampling should be carried out over a time window probably comparable if not longer than the time taken by the full deployment of $I_{s}$ in PMLT.

In fig. $2 \mathrm{~d}$,e the influence of the principal carrier frequency $f_{c}=n p / T$ is highlighted for pulse waves of the same intensity $I$. In particular fig. $2 \mathrm{~d}$ shows that, for fixed $n p$, a signal with lower $f_{c}$ has a higher number of carriers and can then probe a wider range of component depths. In fig. $2 \mathrm{e}$ it is 
shown that prolonging the periodic signal $I_{s}$ with the same value of $f_{c}$ does not affect the number of carriers and their frequency, but the carriers power is increased in the prolonged signal (higher $n p$ ).

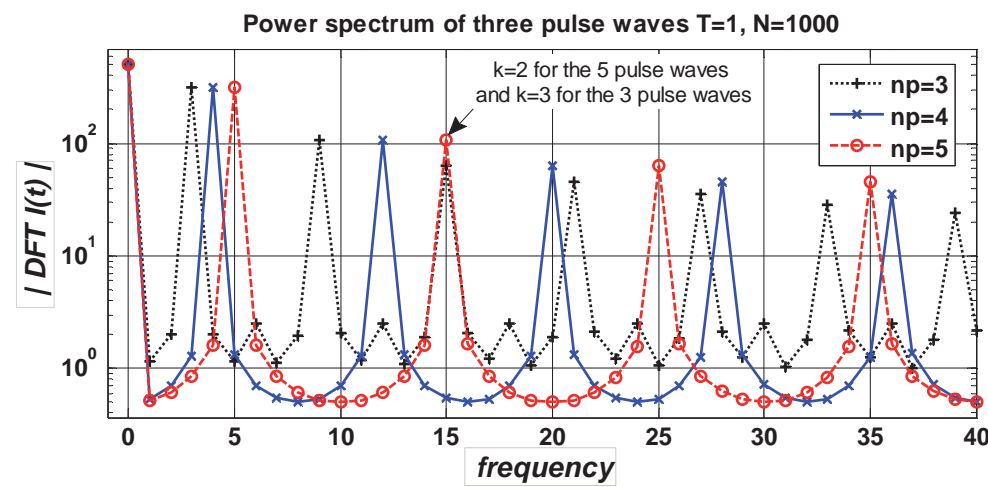

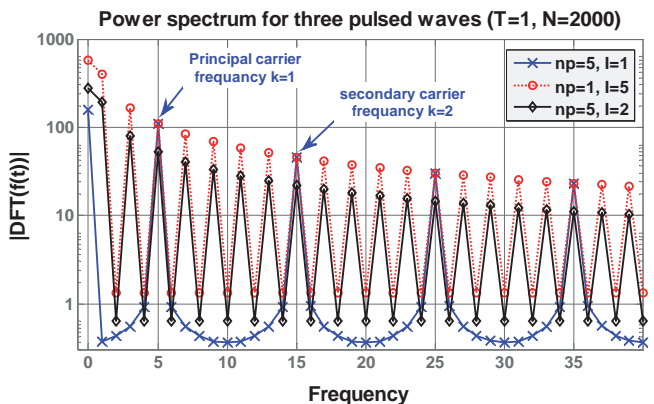

(b)

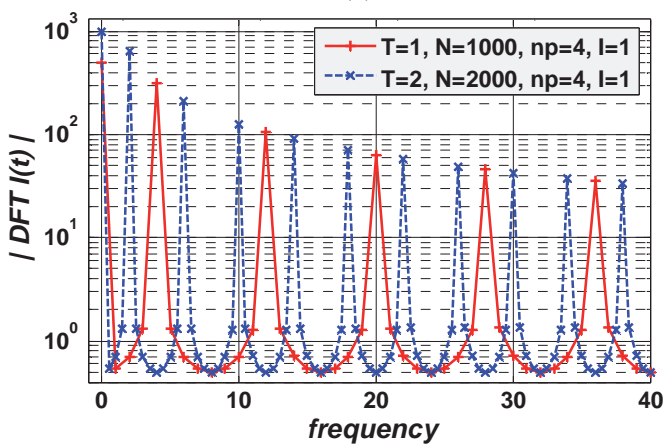

(d)

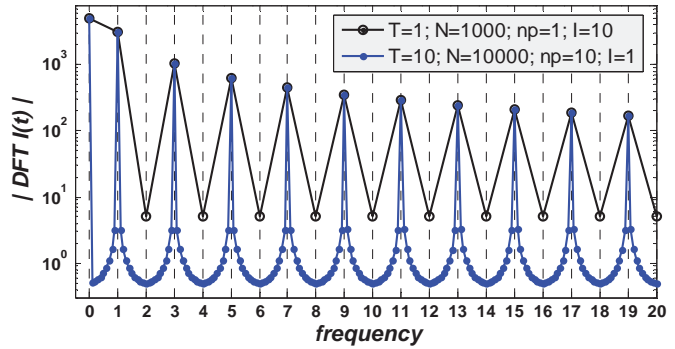

(c)

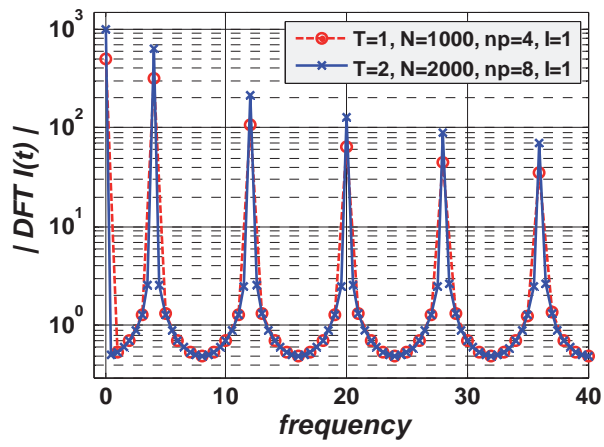

(e)

Fig. 2. Comparison of power spectrums for heat deploying signals $I_{s}$ with different features.

\section{Implementation of a lock-in filtering algorithm for PMLT.}

Lock-in amplifiers are routinely used in Thermoelastic Stress Analysis for filtering out harmonic components of the measured temperature which usually are deeply buried in noise [15]. In this circumstance the harmonic frequency to filter is obtained by providing a periodic reference signal which has the same value of frequency. Commercial hardware or software codes are generally used to perform this lock-in correlation. One such example for a LT application is described in [16]. A simple scheme has recently been proposed [15] which can be easily implemented with common signal and data processing software. This scheme is also implemented in this work and used to find 
the phase shift between a reference signal, e.g. one of the carriers of $I_{s}$, and the harmonic of the measured signal having the same frequency of the reference signal.

A schematic representation of the lock-in correlation is shown in fig. 3. This is implemented in a $\mathrm{Matlab}^{\circledR}$ routine. The frequency to investigate is chosen among the $k$ carrier frequencies of the heat deploying signal. The in-phase and in-quadrature reference signals $F_{k}$ and $G_{k}$ are then built and multiplied to the measured signal $I_{s}$. These two resulting products are then low-pass filtered. This is done by imposing a zero value to the frequency content of the real part of the DFT of $I_{s . .} F_{k}$ and $I s \cdot G k$ and then performing the inverse transform which will result in two constant values: $X$ and $Y$. The phase information is then given by $\phi=\operatorname{atan}(Y / X)$. The procedure can be repeated for different values of $w_{k}$ so that the phase shift is obtained for different probing frequencies. One further advantage of this lock-in scheme is that it will act also as a noise rejecting filter, since all high frequency noise will removed in the low-pass filtering step of the analysis.

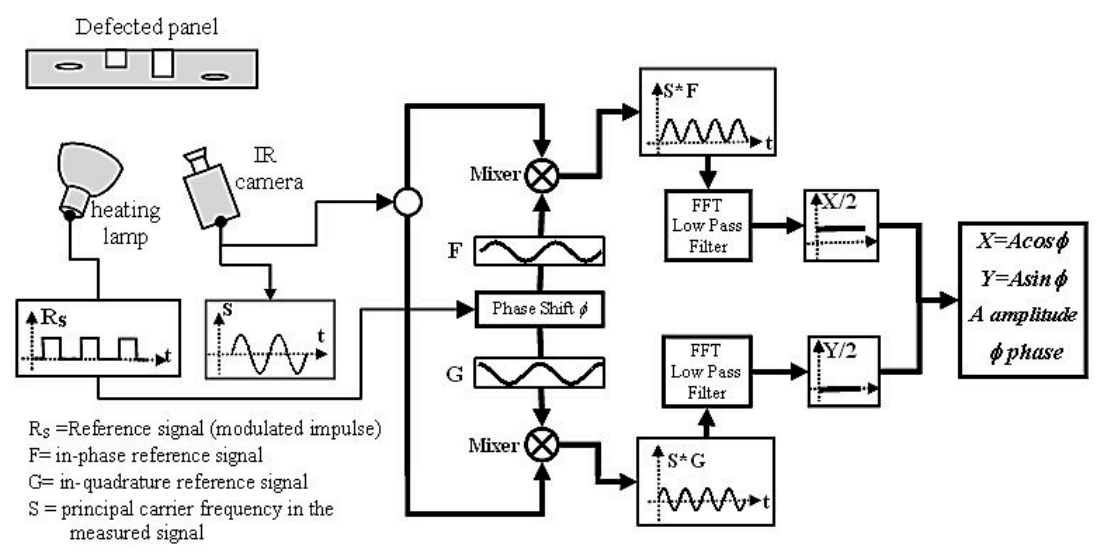

Fig. 3. Scheme of the PMLT set-up and the proposed lock-in correlation signal processing procedure.

\section{Experimental implementation and results.}

In this work the PMLT procedure is implemented to investigate a thick glass fibre reinforced panel whose structural features are peculiar of monolithic composite hulls manufactured for luxury boats and yachts in general. The adoption of a GRP material with a low thermal diffusivity brings some advantages in the implementation of the NDT technique. In fact low frequencies are needed to probe the material. Such frequencies are introduced by a very slow modulation of the heat impulse train which can be controlled manually by just periodically placing a screen panel in front of the optical source to temporarily stop heat deposition. In this way the cost and complexity of the heat delivering system is dramatically reduced.

The investigated sample is a GRP laminate having a thickness of $12 \mathrm{~mm}$. A hand lay-up procedure was used to manufacture the panel whit a cross-ply lay-up obtained by orienting alternate layers at $0^{\circ}$ and $90^{\circ}$. A polyester resin was used requiring a room temperature curing followed by a post cure at 80 degrees for two hours. The unidirectional fabric employed has an areal weight of 400 $\mathrm{g} / \mathrm{m}^{2}$ and the final fibre volume fraction of the panel is about $35 \%$. Three defects were introduced in the central part of the panel by drilling flat bottomed back holes at three different depths from the scanned surface: D1 at $1 / 4, D 2$ at $1 / 2$ and D3 at $3 / 4$ of the laminate thickness. All defects have the same diameter of $20 \mathrm{~mm}$ (see fig. 4a). A peel ply film was placed between the mould and the panel. This allowed to obtain a naturally opaque surface which did not require painting with a matt coat to enhance emissivity or eliminate unduly corrupting reflection during the heat deposition intervals.

The IR set-up employs a low cost linear infrared scanner, Varioscan 3022, with a low thermal resolution (NETD $0.12 \mathrm{~K}$ ). The camera frame rate is about $1.25 \mathrm{~Hz}$ to acquire full-filed temperature 
maps of 360x240 pixels. The camera was remotely controlled with the Irbis v2.0 software and all thermograms imported in Matlab $^{\circledR}$ as 8 bit bitmaps [15]. Each thermogram was also rescaled in Matlab $^{\circledR}$ in order to restore the temperature information. A reflection set-up was adopted, with an external heat source consisting of a $1500 \mathrm{~W}$ halogen lamp placed laterally at about $1 \mathrm{~m}$ from the panel surface.

Results presented in this work are obtained by modulating the heat pulse deposition at 0.00625 $\mathrm{Hz}$ (one period is $160 \mathrm{sec}$ ). Table 1 summarises the values of the PMLT parameters for this application. A cover consisting in a thick panel of polystyrene was placed in front of the lamp at intervals of $80 \mathrm{sec}$ to modulate the square pulses. The cover was manually moved in and out as a shutter in front of the lamp which was switched off during the interval of no heat delivery, to avoid excessive hating of the cover and spurious heat to reach the GRP panel.

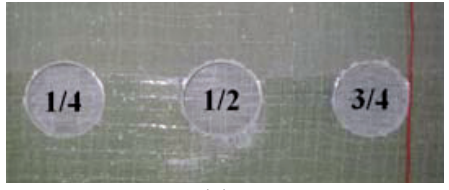

(a)

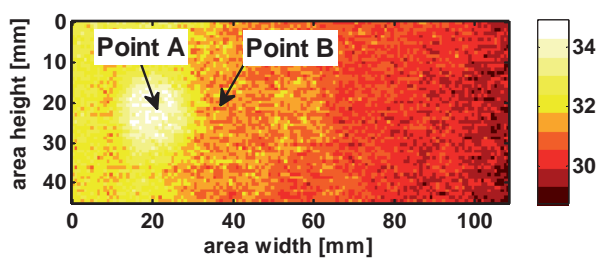

(b)

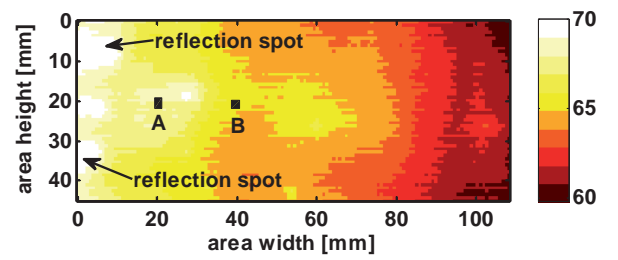

(c)

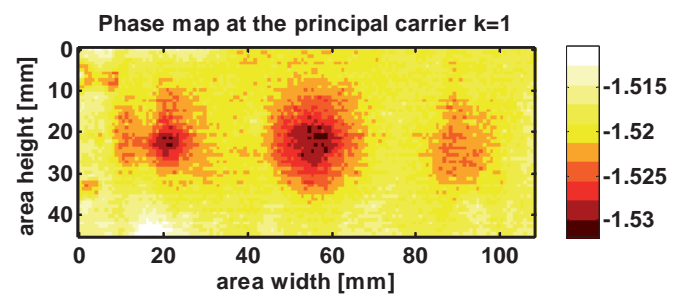

(f)

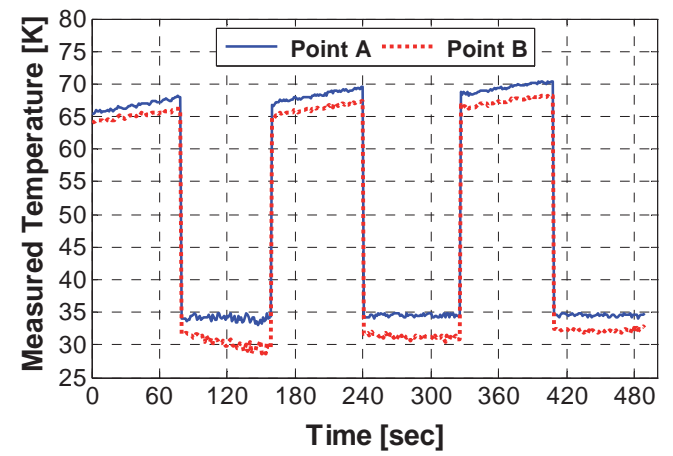

(e)

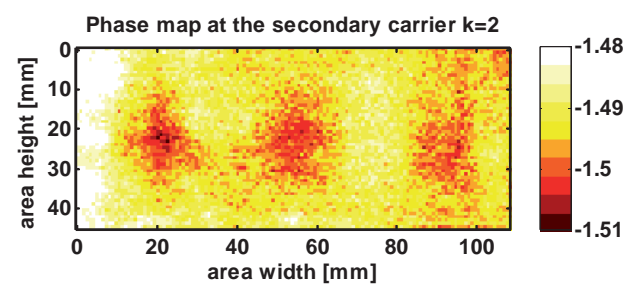

(g)

Fig. 4. Results from the PMLT implementation on the defected GRP panel.

Figure 4 summarises the main findings. Fig. 4a shows a photo of the flat bottom back holes from the face where the holes were drilled. Fig. 4b is a map of the temperature shortly after delivering the first heating pulse (at around $87 \mathrm{sec}$ from start). It is clearly visible that the heat deposition is not uniform over the analysed area. Fig. $4 \mathrm{c}$ shows the temperature distribution after $70 \mathrm{sec}$ from the start, before switching off the lamp after the first heating interval. Some hot spots are observed on the left side of the area due to reflection. This was caused by local defects on the surface finish. On this spots the peel ply did not adhere well leaving a smooth finish which reflects the heat coming from 
the lamp. Fig. 4e shows the temperature versus time plots from point $\mathrm{A}$ (on the location of defect D1) and B (on a sound location between defects D1 and D2). Figures 4f,g show the phase measured form each pixel with the lock-in algorithm described in section 3 . The reference signal for the phase map in fig. 4f was the principal carrier, and the secondary carrier with index $k=2$ was used as reference in fig $4 \mathrm{~g}$. All three defects develop a phase contrast with the surrounding sound area of the material. Furthermore the sound zones, excluding the hot spots, have a more uniform phase distribution compared to the temperature distribution, which is a typical outcome of LT and PPT. Finally the phase contrast for defects D2 and D3 from fig. 4f is higher than from fig. 4g. This is explained because the probing frequency for fig. $f$ is that of the principal carrier, having the lowest value, and hence the ability to probe deeper.

\section{Conclusions}

In this work a low cost Thermal NDE set-up is implemented to investigate a thick glass fibre reinforced composite laminate. A novel Pulse-Modulated Lock-In (PMLT) approach is proposed where a heat impulse is repeatedly applied over a certain time interval in order to modulate the heat deposition at a principal carrier frequency, and introduce, through the square wave shape, secondary carrier frequencies. This carrier frequencies are then used as reference signals for lock-in correlation.

The technique proposed presents some advantages in that: the square wave modulation of the heat source can be easier to implement, several probing frequencies are obtained from one single experiment as in Pulsed-Phase Thermography, and the lock-in signal processing procedure proposed to derive the phase shift maps does not require any hardware or complicate software code and has a beneficial de-noising filtering effect on the harmonics of the measured temperature.

\section{Acknowledgements}

The author would like to acknowledge the support of the University of Palermo through the grant "Progetti d'ateneo ex $60 \% 2007$ ".

\section{References}

1. C. Meola and G.M. Carlomagno, Meas. Sci. Technol., 15, 27 (2004)

2. X.P. Maldague X. Theory and Practice of Infrared Technology for Non-Destructive Testing (Wiley, New York, 2003)

3. C. Ibarra-Castanedo, J.M. Piau, S.Guilbert, N. P. Avdelidis, M. Genest, A. Bendada and X.P.V. Maldagueet al, Research in Nondestructive Evaluation, 20, 1 (2009)

4. M. Susa, X. Maldague, I. Boras, Infrared Physics \& Technology, (2009)

5. S.M. Shepard, J. Hou, J.R. Lhota, J.M. Golden, Optical Engineering 46(5), 051008-1 (2007).

6. H.D. Benitez, H. Loaiza, E. Caicedo, C. Ibarra-Castanedo, A.H. Bendada, X. Maldague, NDT\&E International, 42, 630 (2009).

7. X. Maldague and S. Marinetti, J. Appl. Phys, 79 [5], 2694 (1996)

8. C. Ibarra-Castanedo, Quantitative subsurface defect evaluation by Pulsed Phase thermography: Depth Retrieval with the Phase, PhD thesis. Facultè des sciences et de Gènie Universitè Laval Quèbec, (2005)

9. G. Busse, D.Wu, and W. Karpen, J. Appl. Phys. 71, 3962 (1992)

10. D. Wu and G. Busse, Rev. Gèn. Therm., 37, 693 (1998)

11. W. Bai and B.S. Wong, Meas. Sci. Technol. 12, 142 (2001)

12. G. Giorleo, C. Meola and A. Squillace, Res. Nondestr. Eval., 12, 241 (2000)

13. G. Giorleo, C. Meola, NDT\&E Internatonal 35, 287 (2002)

14. X. Maldague, A. Ziadi, M. Klein, NDT\&E International, 37, 559 (2004)

15. G. Pitarresi, L. D'acquisto, A.M. Siddiolo, J. Strain Analysis 43, 493 (2008)

16. T. Sakagami S. Kubo, Infrared Phys. Technol., 43, 211 (2001) 